\title{
An investigation into the lifestyle and dietary characteristics of normal weight versus overweight and obese shift workers on the island of Ireland
}

\author{
V. O’Brien ${ }^{1}$, F. Nea ${ }^{2}$, L.K. Pourshahidi ${ }^{3}$, J. Kearney ${ }^{2}$, M.B.E. Livingstone ${ }^{3}$ and C.A. Corish ${ }^{4}$ \\ ${ }^{1}$ School of Agriculture and Food Science, University College Dublin, Dublin 4, Ireland, ${ }^{2}$ Dublin Institute of Technology, \\ Kevin Street, Dublin 8, Ireland, ${ }^{3}$ Nutrition Innovation Centre for Food and Health (NICHE), University of Ulster, \\ Coleraine BT52 1SA, UK and ${ }^{4}$ School of Public Health, Physiotherapy and Sports Science, University College Dublin, \\ Dublin 4, Ireland
}

Approximately $17 \%$ of the workforce of the European Union is now engaged in shift work ${ }^{(1)}$. Shift work has been reported to adversely affect the health of workers, leading to a higher risk of overweight/obesity and other chronic diseases ${ }^{(2)}$. This study aimed to compare the lifestyle and dietary characteristics of normal weight and overweight/obese shift workers.

A cross-sectional study was conducted on 1,072 consenting shift workers using a 15-minute telephone questionnaire to establish demographic factors, shift work schedules, current food/drink consumption and snacking habits among shift workers living in Ireland. The questionnaire was developed from Irish qualitative research with shift workers ${ }^{(3,4)}$. Body Mass Index $\left(\mathrm{BMI}, \mathrm{kg} / \mathrm{m}^{2}\right)$ was calculated using self-reported height $(\mathrm{m})$ and weight $(\mathrm{kg})$ measurements, and data were analysed according to WHO BMI categories: Normal weight, BMI $18 \cdot 5-24.9 \mathrm{~kg} / \mathrm{m}^{2} v s$. overweight/obese BMI $\geq 25 \mathrm{~kg} / \mathrm{m}^{2}$.

Within this group of shift workers, $43.7 \%$ were classed as overweight or obese. Univariate analysis indicated that being male, middle/older-aged, consuming $<5$ portions fruit/vegetables per day and having medium-high fried food consumption were associated with overweight/obesity. Smoking and exceeding units of alcohol per week were associated with normal BMI. Following multivariate analysis, being male $[\mathrm{p}<0.001, \mathrm{OR}=2.31,95 \% \mathrm{CI}(1.73-3.09)]$ and middle/older aged were independently associated with overweight/ obese BMI $[\mathrm{p}<0.001, \mathrm{OR}=2.5495 \% \mathrm{CI}(1.85-3.49)$ and $\mathrm{p}<0.001, \mathrm{OR}=2.8995 \% \mathrm{CI}(1.87-4.48)$ respectively]. Dietary behaviours independently associated with overweight/obese BMI included medium-high consumption of fried food [OR $=1.97,95 \%$ CI $(1.48-$ $2.63)]$ and eating less than the recommended number of portions of fruit and vegetables per day [OR $=1 \cdot 38,95 \% \mathrm{CI}(1 \cdot 07-1 \cdot 79)]$. Exceeding recommended units of alcohol per week remained associated with a normal BMI [OR $=0.62,95 \%$ CI $(0 \cdot 38-0 \cdot 99)]$. Smoking was not associated with BMI following multivariate analysis.

\begin{tabular}{|c|c|c|}
\hline & OR & $95 \% \mathrm{CI}$ \\
\hline Male (ref: female) & $2 \cdot 31$ & $1.73-3.09$ \\
\hline \multicolumn{3}{|l|}{ Age (ref: $18-34$ years) } \\
\hline Middle aged ( $34-55$ years) & $2 \cdot 54$ & $1.85-3.49$ \\
\hline Older age ( $>55$ years) & $2 \cdot 89$ & $1.87-4.48$ \\
\hline Smoker (ref: non-smoker) & 0.74 & $0.54-1.02$ \\
\hline Exceed units of alcohol/week (ref: Males $<17$, Females $<11$ ) & $0 \cdot 62$ & $0 \cdot 38-0 \cdot 99$ \\
\hline$<5$ portions of fruit and veg/day (ref: $\geq 5$ ) & $1 \cdot 38$ & $1.07-1.79$ \\
\hline Medium-high fried food consumption (ref: low) & 1.97 & $1.48-2.63$ \\
\hline Low lean meat consumption (ref: medium-high) & $0 \cdot 51$ & $0 \cdot 26-0.98$ \\
\hline
\end{tabular}

This study indicates that shift work is associated with lifestyle and dietary characteristics of shift workers, regardless of BMI. Male and middle/older age shift workers may benefit from targeted dietary and lifestyle advice to protect against overweight and obesity.

This work was supported by safefood, the Food Safety Promotion Board, under Grant No. 10-2013. Ethical approval was obtained from the Dublin Institute of Technology Research Ethics Committee and the study was conducted according to the guidelines laid down in the Declaration of Helsinki.

1. Eurofound (2017) Fifth European Working Conditions Survey. Luxembourg: Publications Office of the European Union.

2. Nea F, Kearney J, Livingstone MBE et al. (2015) Nutr Res Rev 28(2), 143-166.

3. Nea F, Kearney J, Livingstone MBE et al. (2017) J Occup Environ Med 59(12), 1153-1160.

4. Nea F, Kearney J, Livingstone MBE et al. (2018) J Public Health March 13. doi: 10.1093/pubmed/fdy047. [Epub ahead of print] 